Annals of Pure and Applied Mathematics

Vol. 13, No. 1, 2017, 33-40

ISSN: 2279-087X (P), 2279-0888(online)

Published on 13 January 2017

www.researchmathsci.org

DOI: http://dx.doi.org/10.22457/apam.v13n1a4

Annals of

Pure and Applied

Mathematics

\title{
Edge Domination in the Shadow Distance Graph of Some Star Related Graphs
}

\author{
Vijaya Chandra Kumar $U^{1}$ and $R$. Murali ${ }^{2}$
}

${ }^{1}$ School of Physical Science, REVA University, Bengaluru

E-mail: upparivijay@gmail.com

${ }^{2}$ Department of Mathematics, Dr. Ambedkar Institute of Technology, Bengaluru

E-mail: muralir2968@gmail.com

Received 21 December 2016; accepted 31 December 2016

Abstract. Let $G$ be a connected and undirected graph. The shadow graph of $G$, denoted by $D_{2}(G)$ is the graph constructed from $G$ by taking two copies of $G$ namely $G$ itself and $G^{\prime}$ and by joining each vertex $u$ in $G$ to the neighbors of the corresponding vertex $u^{\prime}$ in $G^{\prime}$. Let $D$ be the set of all distance between distinct pairs of vertices in $G$ and let $D_{s}$ (called the distance set) be a subset of $D$. The distance graph of $G$ denoted by $D\left(G, D_{s}\right)$ is the graph having the same vertex as that of $G$ and two vertices $u$ and $v$ are adjacent in $D\left(G, D_{s}\right)$ whenever $d(u, v) \in D_{s}$. In this paper, we determine the edge domination number of the shadow distance graph of the helm graph, the star graph and the subdivision graph of star graph with specified distance sets.

Keywords: Domination set, Edge domination number, Minimal edge dominating set, Shadow distance graph.

AMS Mathematics Subject Classification (2010): 05 C69

\section{Introduction}

The theory of domination in graphs is of vast interest to researchers in graph theory due to its many and varied applications in fields such as optimization, linear algebra, design and analysis of communication networks, military surveillance and social sciences. The study of domination extends to more than four decades and many results have been obtained in the various parameters associated with domination. The concept of edge domination in graphs was introduced by Mitchell and Hedetniemi [2].

By graph $G=(V, E)$ we mean a finite undirected graph without loops and multiple edges. A subset $S$ of $V$ is called a dominating set of $G$ if every vertex not $S$ is 


\section{Vijaya Chandra Kumar U and R Murali}

adjacent to some vertex in $S$. The domination number of $G$ denoted by $\gamma(G)$ is the minimal cardinality taken over all dominating sets of $G$. A subset $F$ of $E$ is called an edge dominating set if each edge in $E$ is either in $F$ or is adjacent to an edge in $F$. An edge dominating set $F$ is called minimal if no proper subset of $F$ is an edge dominating set.

The edge domination number of $G$ denoted by $\gamma^{\prime}(G)$ is the minimum cardinality taken over all edge dominating sets of $G$.

The open neighborhood of an edge $e \in E$ denoted by $N(e)$ is the set of all edges adjacent to $e$ in $G$. If $e=(u, v)$ is an edge in $G$, the degree of $e$ denoted by $\operatorname{deg}(e)$ is given by $\operatorname{deg}(e)=\operatorname{deg}(u)+\operatorname{deg}(v)-2$. The maximum degree of an edge in $G$ is denoted by $\Delta^{\prime}(G)$.

The shadow graph of $G$, denoted by $D_{2}(G)$ is the graph constructed from $G$ by taking two copies of $G$ namely $G$ itself and $G^{\prime}$, and by joining each vertex $u$ in $G$ to the neighbors of the corresponding vertex $u^{\prime}$ in $G^{\prime}$.

Let $D$ be the set of all distance between distinct pairs of vertices in $G$ and let $D_{s}$ (called the distance set) be a subset of $D$. The distance graph of $G$ denoted by $D\left(G, D_{s}\right)$ is the graph having the same vertex as that of $G$ and two vertices $u$ and $v$ are adjacent in $D\left(G, D_{s}\right)$ whenever $d(u, v) \in D_{s}$.

The shadow distance graph of $\mathrm{G}$, denoted by $D_{s d}\left(G, D_{s}\right)$ is constructed from $G$ with the following conditions:

i) consider two copies of $G$ say $G$ itself and $G^{\prime}$

ii) if $u \in V(G)$ (first copy) then we denote the corresponding vertex as $u^{\prime} \in V\left(G^{\prime}\right)$ (second copy)

iii) the vertex set of $D_{s d}\left(G, D_{s}\right)$ is $V(G) \cup V\left(G^{\prime}\right)$

iv) the edge set of $D_{s d}\left(G, D_{s}\right)$ is $E(G) \cup E\left(G^{\prime}\right) \cup E_{d s}$ where $E_{d s}$ is the set of all edges between two distinct vertices $u \in V(G)$ and $v^{\prime} \in V\left(G^{\prime}\right)$ that satisfy the condition $d(u, v) \in D_{s}$ in $\mathrm{G}$.

The Shadow distance graphs $D_{s d}\left(K_{1,3},\{2\}\right)$ and $D_{s d}\left(H_{4},\{3\}\right)$ are illustrated in figure 1 and figure 2 respectively.

The Helm graph denoted by $H_{n}$ is the graph obtained from the $n$-wheel graph $W_{1, n}$ by adjoining a pendant edge at each node of the cycle. We call the edges not in the cycle of $W_{1, n}$ as the spoke edges. 
Edge Domination in the Shadow Distance Graph of Some Star Related Graphs

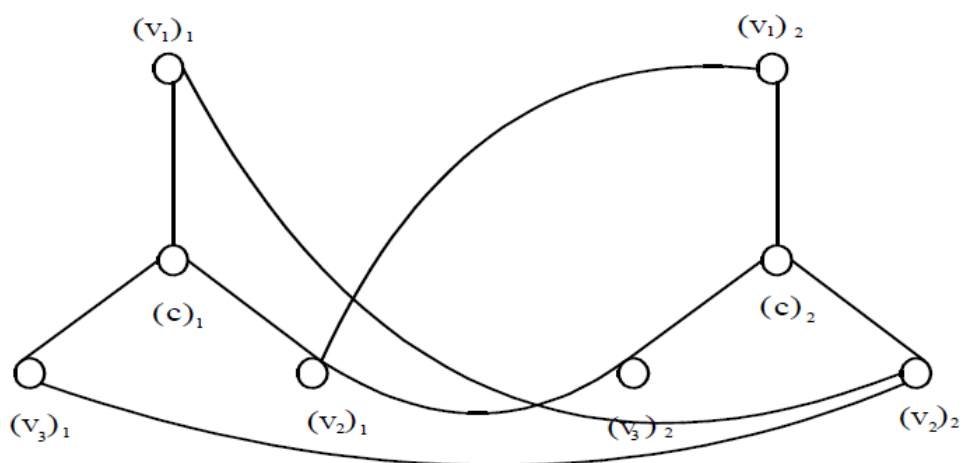

Figure 1: The graph $D_{s d}\left(K_{1,3},\{2\}\right)$

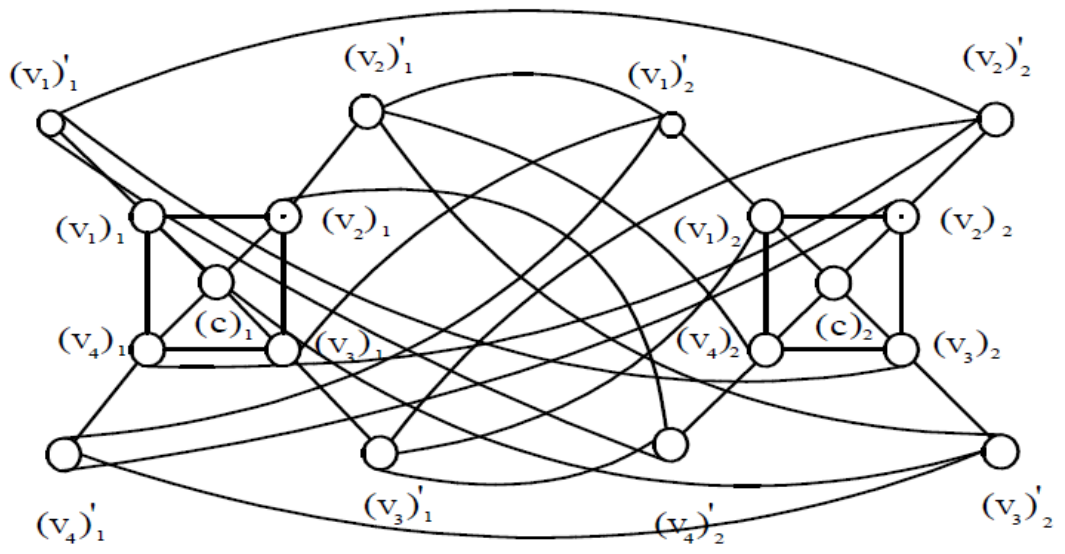

Figure 2: The graph $D_{s d}\left(H_{4},\{3\}\right)$

A star graph is the complete bipartite graph $K_{1, n}$.

If $x=(u, v)$ is edge of $G$, and $\mathrm{w}$ is not a vertex of $G$, then $x$ is subdivided when it is replaced by the edges $(u, w)$ and $(w, v)$. If every edge of $G$ is subdivided, the resulting graph is the subdivision graph $S(G)$.

In the next section we determine the edge domination number of the shadow distance graph of the helm graph and the subdivision graph of the star graph with $D_{s}=\{2\}$ and $\{3\}$ respectively and the star graph with $D_{s}=\{2\}$.

\section{Main results}

We recall the following result related to the edge domination number of a graph . 


\section{Vijaya Chandra Kumar U and R Murali}

Theorem 2.1. [3] An edge dominating set $F$ is minimal if and only if for each edge $e \in G$, one of the following two conditions hold:

i) $\quad N(e) \cap F=\phi$

ii) there exits an edge $e \in E-F$ such that $N(e) \cap F=\{e\}$.

We begin our results with the shadow distance graph associated with the helm graph $H_{n}$.

Theorem 2.2. For $n \geq 3, \gamma^{\prime}\left(D_{s d}\left\{H_{n},\{2\}\right\}\right)=2\left\lceil\frac{n+1}{2}\right\rceil$.

Proof: Consider two copies of $H_{n}$ namely $H_{n}$ itself and $H_{n}^{\prime}$. In the first copy of $H_{n}$, let $\left(v_{1}\right)_{1},\left(v_{2}\right)_{1}, \ldots \ldots \ldots \ldots\left(v_{n}\right)_{1}$ be the vertices of the cycle, let $\left(v_{1}\right)_{1}^{\prime},\left(v_{2}\right)_{1}^{\prime}, \ldots \ldots \ldots \ldots\left(v_{n}\right)_{1}^{\prime}$ be the pendant vertices and $(c)_{1}$ be the central vertex. Let the edges of the cycle be $\left(e_{i}\right)_{1}=\left\{\left\{\left(v_{i}\right)_{1},\left(v_{i+1}\right)_{1}\right\} \cup\left\{\left(v_{1}\right)_{1},\left(v_{i}\right)_{1}\right\}\right\}$ where $1 \leq i \leq n$ and let pendant edges be $\left(e_{p i}\right)_{1}=\left\{\left(v_{i}\right)_{1},\left(v_{i}\right)_{1}^{\prime}\right\}$ where $1 \leq i \leq n$ and spoke edges be $\left(e_{s i}\right)_{1}=\left\{(c)_{1},\left(v_{i}\right)_{1}\right\}$ where $1 \leq i \leq n$. In the second copy, $\left(v_{1}\right)_{2},\left(v_{2}\right)_{2}, \ldots \ldots \ldots \ldots\left(v_{n}\right)_{2}$ be the vertices of the cycle, $\left(v_{1}\right)_{2}^{\prime},\left(v_{2}\right)_{2}^{\prime}, \ldots \ldots \ldots \ldots\left(v_{n}\right)_{2}^{\prime}$ be the pendant vertices and $(c)_{2}$ be the central vertex. Let the edges of the cycle be $\left(e_{i}\right)_{2}=\left\{\left\{\left(v_{i}\right)_{2},\left(v_{i+1}\right)_{2}\right\} \cup\left\{\left(v_{1}\right)_{2},\left(v_{i}\right)_{2}\right\}\right\}$ where $1 \leq i \leq n$ and let pendant edges be $\left(e_{p i}\right)_{2}=\left\{\left(v_{i}\right)_{2},\left(v_{i}^{\prime}\right)_{2}^{\prime}\right\}$ where $1 \leq i \leq n$ and spoke edges be $\left(e_{s i}\right)_{2}=\left\{(c)_{2},\left(v_{i}\right)_{2}\right\}$ where $1 \leq i \leq n$.

Let $G=\left(D_{s d}\left\{H_{n},\{2\}\right\}\right)$.

For $n=3$, the set $F=\left\{e_{4}, e_{s 1}, e_{4}^{\prime}, e_{s 1}^{\prime}\right\}$ is a minimal edge dominating set with minimum cardinality and hence $\gamma^{\prime}(G)=4$.

For $n=4$, the set $F=\left\{e_{4}, e_{8}, e_{s 1}, e_{4}^{\prime}, e_{8}^{\prime}, e_{s 1}^{\prime}\right\}$ is a minimal edge dominating set with minimum cardinality and hence $\gamma^{\prime}(G)=6$.

Let $n \geq 5$

Consider the set $F=\left\{e_{4}, e_{8}, \ldots \ldots e_{4 j}, e_{s 1}, e_{4}^{\prime}, e_{8}^{\prime}, \ldots . . e_{4 j}^{\prime}, e_{s 1}^{\prime}\right\}$ where $1 \leq j \leq\left\lfloor\frac{n-1}{2}\right\rfloor$.

This set $F$ is a minimal edge dominating set since for any edge $e_{i} \in F, F-\left\{e_{i}\right\}$ is not an edge dominating set for $N\left(e_{i}\right)$ in $G$. Hence any set containing edges less than that of $F$ cannot be a dominating set of $G$. Further, $\Delta^{\prime}(G)=3 n+1$ which implies that an edge of $G$ can dominate at most $3 n+2$ distinct edges including itself and each of 
Edge Domination in the Shadow Distance Graph of Some Star Related Graphs the remaining edges can dominate less than $3 n+1$ edges of $G$. Therefore, any set containing the edges less that in $F$ cannot be an edge dominating set of $G$.

This implies that the set $F$ described above is of minimum cardinality and since $|F|=2\left\lceil\frac{n+1}{2}\right\rceil$, it follows that $\gamma^{\prime}\left(D_{s d}\left\{H_{n},\{2\}\right\}\right)=2\left\lceil\frac{n+1}{2}\right\rceil$.

Hence the proof.

Theorem 2.3. For $n \geq 3$, $\gamma^{\prime}\left(D_{s d}\left\{H_{n},\{3\}\right\}\right)=2\left\lceil\frac{2 n-1}{2}\right\rceil$.

Proof: Let $G=\left(D_{s d}\left\{H_{n},\{3\}\right\}\right)$ The vertex set and edge set of $G$ are as in theorem 2.2.

For $n=3$, the set $F=\left\{e_{1}, e_{4}, e_{6}, e_{1}^{\prime}, e_{4}^{\prime}, e_{6}^{\prime}\right\}$ is minimal a edge dominating set with minimum cardinality and hence $\gamma^{\prime}(G)=6$.

Let $n \geq 4$.

\section{Consider the set}

$F=\left\{e_{1}, e_{3}, e_{5}, \ldots . . . e_{2 j-1}, e_{1}^{\prime}, e_{3}^{\prime}, e_{5}^{\prime}, \ldots . . e_{2 j-1}^{\prime}\right\}$ where $1 \leq j \leq\left\lfloor\frac{2 n-1}{2}\right\rfloor$.

This set $F$ is a minimal edge dominating set since for any edge $e_{i} \in F, F-\left\{e_{i}\right\}$ is not an edge dominating set for $N\left(e_{i}\right)$ in $G$. Hence any set containing edges less than that of $F$ cannot be a dominating set of $G$. Further, $\Delta^{\prime}(G)=2 n$ which implies that an edge of $G$ can dominate at most $2 n+1$ distinct edges including itself and each of the remaining edges can dominate less than $2 n$ edges of $G$. Therefore, any set containing the edges less that in $F$ cannot be an edge dominating set of $G$.

This implies that the set $F$ described above is of minimum cardinality and since $|F|=2\left\lceil\frac{2 n-1}{2}\right\rceil$, it follows that $\gamma^{\prime}\left(D_{s d}\left\{H_{n},\{3\}\right\}\right)=2\left\lceil\frac{2 n-1}{2}\right\rceil$.

Hence the proof.

For the star graph $K_{1, n}$, we have the following result.

Theorem 2.4. For $n \geq 4, \gamma^{\prime}\left(D_{s d}\left\{K_{1, n},\{2\}\right\}\right)=2\left\lceil\frac{n-1}{2}\right\rceil$.

Proof: Consider two copies of $K_{1, n}$ namely $K_{1, n}$ itself and $K_{1, n}^{\prime}$. In the first copy of $K_{1, n}$, let $\left(v_{1}\right)_{1},\left(v_{2}\right)_{1}, \ldots \ldots \ldots \ldots\left(v_{n}\right)_{1}$ be the pendant vertices and $(c)_{1}$ be the central vertex and let the edges be $\left(e_{i}\right)_{1}=\left\{(c)_{1},\left(v_{i}\right)_{1}\right\}$ where $1 \leq i \leq n$. In the second copy $K_{1, n}^{\prime}$, 


\section{Vijaya Chandra Kumar U and R Murali}

$\left(v_{1}\right)_{2},\left(v_{2}\right)_{2}, \ldots \ldots \ldots \ldots\left(v_{n}\right)_{2}$ be the pendant vertices and $(c)_{2}$ be the central vertex and let the edges be $\left(e_{i}^{\prime}\right)_{2}=\left\{(c)_{2},\left(v_{i}\right)_{2}\right\}$ where $1 \leq i \leq n$.

Let $G=\gamma^{\prime}\left(D_{s d}\left\{K_{1, n},\{2\}\right\}\right)$

For $n=4$ and $n=5$, the set $F=\left\{e_{2}, e_{14^{\prime}}, e_{6}, e_{2}^{\prime}, e_{14}^{\prime}\right\}$ is a minimal edge dominating set with minimum cardinality and hence $\gamma^{\prime}(G)=4$.

Let $n \geq 6$.

Consider the set

$F=\left\{e_{2}, e_{(i)(i+3)^{\prime}}, e_{(2 j+3)(2 k+4)^{\prime}}, e_{2}^{\prime}, e_{(i)^{\prime}(i+3)}^{\prime}, e_{(2 k+3)^{\prime}(2 k+4)}^{\prime}\right\}$ where $i=1,1 \leq j, k \leq\left\lfloor\frac{n-4}{2}\right\rfloor$

This set $F$ is a minimal edge dominating set since for any edge $e_{i} \in F, F-\left\{e_{i}\right\}$ is not an edge dominating set for $N\left(e_{i}\right)$ in $G$. Hence any set containing edges less than that of $F$ cannot be a dominating set of $G$. Further, $\Delta^{\prime}(G)=n(n-1)$ which implies that an edge of $G$ can dominate at most $n(n-1)+1$ distinct edges including itself and each of the remaining edges can dominate less than $n(n-1)$ edges of $G$. Therefore, any set containing the edges less that in $F$ cannot be an edge dominating set of $G$.

This implies that the set $F$ described above is of minimum cardinality and since $|F|=2\left\lceil\frac{n-1}{2}\right\rceil$, it follows that $\gamma^{\prime}\left(D_{s d}\left\{K_{1, n},\{2\}\right\}\right)=2\left\lceil\frac{n-1}{2}\right\rceil$.

Hence the proof.

For the subdivision graph of the star graph $S\left(K_{1, n}\right)$, we have the following results.

Theorem 2.5. For $n \geq 3$, $\gamma^{\prime}\left(D_{s d}\left\{S\left(K_{1, n}\right),\{2\}\right\}\right)=2 n$.

Proof: Consider two copies of $S\left(K_{1, n}\right)$ namely $S\left(K_{1, n}\right)$ itself and $S^{\prime}\left(K_{1, n}\right)$. In the first copy of $S\left(K_{1, n}\right)$, let $\left(v_{1}\right)_{1},\left(v_{2}\right)_{1}, \ldots \ldots \ldots \ldots\left(v_{n}\right)_{1}$ be the pendant vertices and $\left(l_{1}\right)_{1},\left(l_{2}\right)_{1}, \ldots \ldots \ldots \ldots\left(l_{n}\right)_{1}$ be the sub division vertices and let $(c)_{1}$ be the central vertex which is adjacent to $\left(l_{i}\right)_{1}$, where $1 \leq i \leq n$. Let the edges be $e_{i}=\left\{\left(\left(v_{i}\right)_{1},\left(l_{i}\right)_{1}\right) \cup\left((c)_{1},\left(l_{i}\right)_{1}\right)\right\}$ where $1 \leq i \leq n$. In the second copy $S^{\prime}\left(K_{1, n}\right)$, let $\left(v_{1}\right)_{2},\left(v_{2}\right)_{2}, \ldots \ldots \ldots \ldots\left(v_{n}\right)_{2}$ be the pendant vertices and $\left(l_{1}\right)_{2},\left(l_{2}\right)_{2}, \ldots \ldots \ldots \ldots . .\left(l_{n}\right)_{2}$ be the sub division vertices and let $(c)_{2}$ be the central vertex which is adjacent to $\left(l_{i}\right)_{2}$, where $1 \leq i \leq n$. Let the edges be $e_{i}^{\prime}=\left\{\left(\left(v_{i}\right)_{2},\left(l_{i}\right)_{2}\right) \cup\left((c)_{2},\left(l_{i}\right)_{2}\right)\right\}$ where $1 \leq i \leq n$.

Let $G=\gamma^{\prime}\left(D_{s d}\left\{S\left(K_{1, n}\right),\{2\}\right\}\right)$ 
Edge Domination in the Shadow Distance Graph of Some Star Related Graphs

Let $n \geq 3$.

Consider the set $F=\left\{e_{2}, e_{4^{\prime}}, e_{6}, \ldots . . e_{2 i}, e_{2}^{\prime}, e_{4}^{\prime}, e_{6}^{\prime}, \ldots . . e_{2 i}^{\prime}\right\}$ where $1 \leq i \leq n$.

This set $F$ is a minimal edge dominating set since for any edge $e_{i} \in F, F-\left\{e_{i}\right\}$ is not an edge dominating set for $N\left(e_{i}\right)$ in $G$. Hence any set containing edges less than that of $F$ cannot be a dominating set of $G$. Further, $\Delta^{\prime}(G)=3 n+5$ which implies that an edge of $G$ can dominate at most $3 n+6$ distinct edges including itself and each of the remaining edges can dominate less than $3 n+5$ edges of $G$. Therefore, any set containing the edges less that in $F$ cannot be an edge dominating set of $G$.

This implies that the set $F$ described above is of minimum cardinality and since $|F|=2 n$, it follows that $\gamma^{\prime}\left(D_{s d}\left\{S\left(K_{1, n}\right),\{2\}\right\}\right)=2 n$.

Hence the proof.

Theorem 2.6. For $n \geq 3, \gamma^{\prime}\left(D_{s d}\left\{S\left(K_{1, n}\right),\{3\}\right\}\right)=2 n$.

Proof: Let $G=\gamma^{\prime}\left(D_{s d}\left\{S\left(K_{1, n}\right),\{3\}\right\}\right)$. The vertex set and edge set of $G$ are as in theorem 2.5.

Let $n \geq 3$.

Consider the set $F=\left\{e_{2}, e_{4^{\prime}}, e_{6}, \ldots . . e_{2 i}, e_{2}^{\prime}, e_{4}^{\prime}, e_{6}^{\prime}, \ldots . . e_{2 i}^{\prime}\right\}$ where $1 \leq i \leq n$.

This set $F$ is a minimal edge dominating set since for any edge $e_{i} \in F, F-\left\{e_{i}\right\}$ is not an edge dominating set for $N\left(e_{i}\right)$ in $G$. Hence any set containing edges less than that of $F$ cannot be a dominating set of $G$. Further, $\Delta^{\prime}(G)=2 n+3$ which implies that an edge of $G$ can dominate at most $2 n+4$ distinct edges including itself and each of the remaining edges can dominate less than $2 n+3$ edges of $G$. Therefore, any set containing the edges less that in $F$ cannot be an edge dominating set of $G$.

This implies that the set $F$ described above is of minimum cardinality and since $|F|=2 n$, it follows that $\gamma^{\prime}\left(D_{s d}\left\{S\left(K_{1, n}\right),\{3\}\right\}\right)=2 n$.

Hence the proof.

Acknowledgements. The first author is thankful to the Management and staff of the School of Physical Science (Mathematics), REVA University, Bengaluru. The authors are also thankful to the Management and Research Centre, Department of Mathematics, Dr. Ambedkar Institute of Technology, Bengaluru.

\section{REFERENCES}

1. S.K.Vaidya and R.M.Pandit, Edge domination in some path and cycle related graphs, ISRN Discrete Mathematics, Volume 2014 (2014), 1-5. 


\section{Vijaya Chandra Kumar U and R Murali}

2. S.T.Hedetniemi and R.C.Laskar, Bibliography on domination in graphs and some basic definitions of domination parameters, Discrete Mathematics, Vol. 86, No.1-3 (1990) 257-277.

3. V.R.Kulli, Theory of domination in graphs, Vishwa International Publications, 2013.

4. S.R.Jayaram, Line domination in graphs, Graphs and Combinatorics, Vol. 3, No. 4 (1987) 357-363.

5. Frank Harary, Graph Theory, Addison-Wesley, 1969.

6. S.Chandra Kumar and T.Nicholas, b-coloring in Square of Cartesian Product of Two Cycles, Annals of Pure and Applied Mathematics, 1(2) (2012) 131-137.

7. Kalyani Das and Madhumangal Pal, An Optimal Algorithm to Find a Minimum 2neighbourhood Covering Set on Cactus Graphs, Annals of Pure and Applied Mathematics, 2(1) (2012) 45-59.

8. Kalyani Das, Some Algorithms on Cactus Graphs, Annals of Pure and Applied Mathematics, 2(2) (2012) 114-128.

9. S.Sriram, D.Ranganayakulu, Ibrahim Venkat and K.G.Subramanian, On Eccentric Graphs of Broom Graphs, Annals of Pure and Applied Mathematics, 5(2) (2014) 146-152. 